\title{
Nuclear resonant forward scattering of synchrotron radiation by randomly oriented iron complexes which exhibit nuclear Zeeman interaction
}

\author{
M. Haas and E. Realo \\ Institute of Physics, Estonian Academy of Sciences, EE-2400 Tartu, Estonia \\ H. Winkler, W. Meyer-Klaucke, and A. X. Trautwein \\ Institut für Physik, Medizinische Universität zu Lübeck, D-23538 Lübeck, Germany \\ O. Leupold and H. D. Rüter \\ II. Institut für Experimentalphysik, Universität Hamburg, D-22761 Hamburg, Germany
}

(Received 14 July 1997)

\begin{abstract}
An expression for the amplitude of a pulse of synchrotron radiation (SR) coherently scattered in forward direction by a randomly oriented Mössbauer absorber is derived from the theory of $\gamma$ optics. It is assumed that the hyperfine splittings present in the Mössbauer nuclei can be described in the framework of the spinHamiltonian formalism. In the general case of a thick Mössbauer sample, which consists of randomly oriented paramagnetic iron-containing molecules (for example, a frozen solution of a ${ }^{57} \mathrm{Fe}$ protein) in an applied magnetic field, the response of this sample on an incident monochromatic and fully polarized SR beam cannot be given analytically because of the integrations involved. The way to evaluate nuclear forward-scattering spectra for this general case numerically is outlined and results of calculations with a corresponding program package called SYNFOS are shown and compared with experimental results obtained by measurements of the high-spin iron (II) "picket-fence", porphyrin $\left[\mathrm{Fe}\left(\mathrm{CH}_{3} \mathrm{COO}\right) \mathrm{TP}_{\text {piv }} \mathrm{P}\right]^{-}$in an applied field of $6 \mathrm{~T}$. [S0163-1829(97)02545-9]
\end{abstract}

\section{INTRODUCTION}

Nuclear resonant forward scattering (NFS) of synchrotron radiation $^{1,2}$ is a tool for studying the electronic structure of Mössbauer atoms which is of major interest in many fields of research. In particular, investigations of paramagnetic macromolecules with mono- or oligonuclear iron centers, the behavior of which is conveniently described in terms of the spin-Hamiltonian formalism, are expected to benefit from this kind of spectroscopy, because additional external parameters like polarization and time structure of the probing radiation can be introduced into the protocol of applied experimental conditions. In order to gain access to the wealth of information which can be obtained from time-resolved scattering experiments, especially on randomly oriented scatterers (frozen solution or polycrystals of an iron-containing protein), a theoretical approach is needed to form the basis for a computer code by which the experimental spectra can be simulated. Theoretical approaches and computer codes have been developed for monocrystals (including the Bragg scattering case) in Ref. 3 and for grazing incidence in Ref. 4. However, for randomly oriented molecules, especially when the molecular electronic and magnetic structure requires a tensor description (electric field gradient tensor and magnetic hyperfine coupling tensor), none of the details entering a proper computer simulation of NFS have been provided so far.

In the present paper the case of randomly oriented scatterers with electric and/or magnetic hyperfine splitting of nuclear levels is considered, with the propagation of radiation in samples being described in terms of Mössbauer optics. The foundation of such an optical approach has been given in Refs. 5-7 where the multiple scattering of $\gamma$ radiation is discussed in detail and the key role of coherent forward scattering is shown. In the following the basic formulas of our theoretical approach are presented as well as the results of calculations performed with a FORTRAN program package which has been given the name SYNFOS, an acronym formed from the term "syn' "chrotron radiation "for" ward "s" cattering.

\section{BASIC FORMULAS}

\section{A. Randomly oriented Mössbauer scatterer}

The subject of this paper is a scatterer consisting of randomly oriented iron complexes, placed in an external field $\vec{B}$. The complexes contain Mössbauer nuclei, which are assumed to be homogeneously distributed. Further on, the case of paramagnetic ions, characterized by an effective electron spin $S$, will be considered predominantly. The electronic ground states of such ions form an isolated multiplet, described by a Hamiltonian of the form ${ }^{8}$

$$
H_{\mathrm{el}}=\hat{\vec{S}} \cdot \tilde{D} \cdot \hat{\vec{S}}+\beta_{e} \hat{\vec{S}} \cdot \widetilde{g} \cdot \vec{B}
$$

with eigenfunctions and eigenvalues $\Phi_{l}^{(\mathrm{el})}$ and $\varepsilon_{l}$ $(l=1, \ldots, 2 S+1)$. In Eq. (1), $\widetilde{D}$ and $\widetilde{g}$ are tensors describing electronic spin-orbital and Zeeman interactions, respectively. $\hat{\vec{S}}$ is the effective spin operator, and $\beta_{e}$ is the Bohr magneton.

Mössbauer nuclei are characterized by the spin $I_{0}$ of the ground and $I_{e}$ of the excited state, by the width $\Gamma$ of single zero-phonon absorption lines and by the frequency $\omega_{r}$ of the 
Mössbauer transition in the absence of hyperfine interactions. The following treatment assumes that the splitting of the electronic levels is considerably larger than the hyperfine energies. Under this assumption the hyperfine splitting of the ground level (0) and the excited level $(e)$ of a Mössbauer nucleus is given by the Hamiltonians $H^{(0)}$ and $H^{(e)}$, respectively:

$$
H^{(0)(e)}=\vec{s} \cdot \widetilde{A}^{(0)(e)} \cdot \hat{\vec{I}}-\beta_{n} g_{n}^{(0)(e)} \hat{\vec{I}} \cdot \vec{B}+\hat{\vec{I}} \cdot \widetilde{V}^{(0)(e)} \cdot \hat{\vec{I}} .
$$

Here $\hat{\vec{I}}$ is the nuclear spin operator, and the tensors $\widetilde{A}$ and $\widetilde{V}$ describe the magnetic hyperfine interaction and the nuclear quadrupole interaction. The second term in Eq. (2) is the nuclear Zeeman interaction term, where $g_{n}$ is the nuclear $g$ factor and $\beta_{n}$ the nuclear magneton. When the transitions between the electronic multiplet substates are slow compared to the nuclear precession frequencies (slow relaxation limit), the vectors $\vec{s}_{l}$ are determined by

$$
\vec{s}_{l}=\left\langle\phi_{l}^{(\mathrm{el})}|\hat{\bar{S}}| \Phi_{l}^{\mathrm{el}}\right\rangle .
$$

In the case of fast relaxation, only the thermal average

$$
\vec{s}=\sum_{l} w_{l} \vec{s}_{l}
$$

is observable, where the weight of the $l$ th electronic state is

$$
w_{l}=\exp \left(-\varepsilon_{l} / k_{B} T\right)\left[\sum_{l^{\prime}} \exp \left(-\varepsilon_{l^{\prime}} / k_{B} T\right)\right]^{-1},
$$

$T$ is the temperature of the sample, and $k_{B}$ is the Boltzmann constant.

The form of the eigenfunctions of the Hamiltonians (1) and (2) is especially simple in the molecular frame of reference. In this frame the eigenfunctions $\Phi_{l}^{(\mathrm{el})}$ as well as the energies $\varepsilon_{l}$ depend on the polar and azimuthal angle $\theta$ and $\varphi$, respectively, of the external field $\vec{B}\left[\Phi_{l}^{(\mathrm{el})} \equiv \Phi_{l}^{(\mathrm{el})}(\theta, \varphi), \varepsilon_{l}\right.$ $\left.\equiv \varepsilon_{l}(\theta, \varphi)\right]$. In the slow-relaxation case, the nuclear multiplet wave functions $\left(\Phi_{i}^{(0)}\right.$ and $\left.\Phi_{f}^{(e)}\right)$ and the corresponding energies $\left(\hbar \omega_{i}\right.$ and $\left.\hbar \omega_{f}\right)$ depend also on the electronic state of the ion $\left[\hbar \omega_{i(f)} \equiv \hbar \omega_{i(f)}(l ; \theta, \varphi)\right]$. Further, we consider mostly the case of a significant hyperfine splitting of the nuclear levels, when the sample of randomly oriented complexes is characterized by a band of resonant absorptions of a width $\Delta$ with $\Delta \gg \Gamma$. ${ }^{9}$ This band has a complicated structure and cannot be described by any inhomogeneously broadened single line.

\section{B. Synchrotron radiation pulses in a Mössbauer scatterer}

The pulse of synchrotron radiation (SR) incident on the scatterer as defined in the previous section is described by the electric field of the radiation:

$$
\vec{E}_{0}(z, t)=\vec{e}_{0}\left(t-z / c_{0}\right) \exp \left[-i \omega_{0}\left(t-z / c_{0}\right)\right], \quad z \leqslant 0 .
$$

$c_{0}$ is the speed of light in vacuum. The $\gamma$ quanta propagate in the direction of the $z$ axis, normal to the surface of the Mösbauer sample. It is assumed that the radiation is fully linear polarized along the $x$ axis $\left[\vec{e}_{0}=\left(e_{0}, 0,0\right)\right]$. This direction is also labeled by $\vec{\sigma}$ in the following.
Transient processes in the scatterer modify the incident SR pulse. We are interested in the time range after $t_{0}$, when the short incident pulse is terminated $\left[E_{0}(d, t) \rightarrow 0, t \gg t_{0}\right]$ and beyond which the evolution of the transmitted pulse is determined by forward-scattered radiation alone. In this time range the transmitted radiation field can be represented as

$$
\begin{aligned}
E_{\alpha}(z, t)= & (2 \pi)^{-1} \int_{-\infty}^{\infty} d \omega \exp \left[-i \omega\left(t-z / c_{0}\right)\right] \\
& \times\left[\widetilde{R}_{\alpha x}(\omega, d)-\widetilde{\delta}_{\alpha x}\right] \chi_{0},
\end{aligned}
$$

where $\alpha=x, y$ and $d$ is the thickness of the sample with $z$ $\geqslant d$. In Eq. (7) the components of the tensor $\widetilde{R}(\omega, d)$ represent the response of the scatterer on a stationary beam of monochromatic Mössbauer radiation, and $\widetilde{\delta}$ is the unit tensor. Also, the approximate relation $e_{0}(\tau) \approx \delta(\tau) \int_{-\infty}^{\infty} e_{0}(t) d t$ $=\chi_{0} \delta(\tau)$ has been used. ${ }^{10}$ It should be noted that Bragg diffraction is neglected in randomly oriented samples.

A semiclassical optical theory, describing the transformation of a monochromatic beam of Mössbauer radiation in resonant media, has been developed in Refs. 5-7. This theory can be modified to derive the explicit form of $\widetilde{R}_{\alpha \beta}$ for the cases of randomly oriented samples:

$$
\widetilde{R}_{\alpha \beta}(\omega, d)=\sum_{j=1,2} \tilde{\rho}_{\alpha \beta}^{(j)} \exp \left\{\left(i \omega d / c_{0}\right)\left[n^{(j)}(\omega)-1\right]\right\}
$$

In Eq. (8), $j$ indicates the two elliptically polarized plane waves arising in the medium due to the birefringence phenomenon. The refractive indices $n^{(j)}(\omega)$ of these waves are expressed as

$$
n^{(j)}(\omega)=n_{\mathrm{el}}(\omega)+\left[U^{(j)}(\omega, d)\right]\left(c_{0} / \omega d\right),
$$

where $n_{\mathrm{el}}(\omega)$ accounts for the polarization of the electronic subsystem. The contribution of the resonant Mössbauer nuclei $U^{(j)}(\omega, d)$ is determined by the average elastic forwardscattering amplitudes $\bar{f}_{\alpha \beta} \equiv \bar{f}_{\alpha \beta}(\omega)$ of a single nucleus:

$$
U^{(j)}(\omega, d)=d\left\{\bar{f}_{x x}+\bar{f}_{y y}+(-1)^{j}\left[\left(\bar{f}_{x x}-\bar{f}_{y y}\right)^{2}+4 \bar{f}_{x y} \bar{f}_{x y}\right]^{1 / 2}\right\} .
$$

The subscripts $\alpha$ and $\beta$ indicate the polarization of the absorbed and the emitted photons $(\alpha, \beta=x, y)$. Also, the nonzero components of the tensor $\tilde{\rho}^{(j)}$ in Eq. (8) are determined by the scattering amplitudes $\bar{f}_{\alpha \beta}$ :

$$
\begin{aligned}
\tilde{\rho}_{\alpha \beta}^{(j)}= & (-1)^{j}\left(c_{0} / \omega\right)\left\{\widetilde{\delta}_{\alpha \beta}\left[\left(n^{(j)}-n_{\mathrm{el}}\right)\left(\omega / c_{0}\right)\right]\right. \\
& \left.-2 f_{\alpha \beta}\right\} /\left(n^{(1)}-n^{(2)}\right) .
\end{aligned}
$$

To arrive at an explicit form for $U^{(j)}(\omega)$ and $\tilde{\rho}_{\alpha \beta}^{(j)}$, the averaged forward-scattering amplitudes $\bar{f}_{\alpha \beta}$ are expressed as 


$$
\begin{aligned}
\bar{f}_{\alpha \beta}(\omega)= & \left(8 \pi^{2}\right)^{-1}(b / d) \int_{0}^{\pi} \sin \theta^{\prime} d \theta^{\prime} \int_{0}^{2 \pi} \int_{0}^{2 \pi} d \varphi^{\prime} d \psi \\
& \times \sum_{l} w_{l} \sum_{\mu, \mu^{\prime}= \pm 1,0}(-1)^{\mu} \\
& \times \Pi_{\alpha,-\mu}\left(\psi, \theta^{\prime}, \varphi^{\prime}\right) \Pi_{\beta \mu^{\prime}}\left(\psi, \theta^{\prime}, \varphi^{\prime}\right) \\
& \times \sum_{n} A_{\mu \mu^{\prime}}^{(n)} /\left(\omega_{r}-\omega+\omega_{n}-i \Gamma / 2\right) .
\end{aligned}
$$

In Eq. (12), $n$ represents the $\left(2 I_{0}+1\right) \times\left(2 I_{e}+1\right)$ Mössbauer transitions in the nucleus, i.e., $n=(i, f), \omega_{n}=\omega_{f}-\omega_{i}$, and the shorthand notation $b$ is defined as ${ }^{5-7,11}$

$$
\begin{aligned}
b= & \left\{c_{0}^{2} d \exp \left(-2 W_{D}\right) \Gamma /\left[(1+\alpha) V_{0} \omega^{2}\right]\right\} \\
& \times\left[\left(2 I_{e}+1\right) / 2\left(2 I_{0}+1\right)\right],
\end{aligned}
$$

where $V_{0}^{-1}$ is the density of Mössbauer nuclei, $\exp \left(-2 W_{D}\right)$ is the Debye-Waller factor, and $\alpha$ is the conversion coefficient. The tensors $A_{\mu \mu^{\prime}}^{(n)}$ in Eq. (12) are determined by the transition matrix elements and take in case of $M 1$ transitions: e.g., for ${ }^{57} \mathrm{Fe}$, the explicit form ${ }^{5-7,11}$

$$
\begin{aligned}
A_{\mu \mu^{\prime}}^{(n)}= & {\left[3 /\left(2 I_{e}+1\right)\right] \sum_{m_{e} m_{e}^{\prime}} \sum_{m_{0} m_{0}^{\prime}}\left(I_{0} m_{0} ; 1, \mu \mid I_{e} m_{e}\right) } \\
& \times\left(I_{0} m_{0}^{\prime} ; 1, \mu^{\prime} \mid I_{e} m_{e}^{\prime}\right) C_{i m_{0}}^{*} C_{i m_{0}^{\prime}} C_{f m_{e}^{\prime}}^{*} C_{f m_{e}},
\end{aligned}
$$

where $C_{i m_{0}}$ and $C_{f m_{e}}$ are coefficients of expansion of the nuclear multiplet wave functions $\Phi_{i}^{(0)}$ and $\Phi_{f}^{(e)}$ in the series of the eigenfunctions of the $\hat{I}_{z}$ operator. Finally, the matrices $\Pi_{\alpha \mu}\left(\psi, \theta^{\prime}, \varphi^{\prime}\right)$ in Eq. (14) have the following components:

$$
\begin{gathered}
\Pi_{x \mu}=(1 / \sqrt{2})\left(D_{1 \mu}^{1}-D_{-1 \mu}^{1}\right) \quad \text { and } \\
\Pi_{y \mu}=(i / \sqrt{2})\left(D_{1 \mu}^{1}+D_{-1 \mu}^{1}\right) .
\end{gathered}
$$

Here $D_{\mu \mu^{\prime}}^{1} \equiv D_{\mu \mu^{\prime}}^{1}\left(\psi, \theta^{\prime}, \varphi^{\prime}\right)$ are the Wigner functions which couple the transition matrix elements, calculated in the laboratory and molecular reference systems, respectively, and $\left(\psi, \theta^{\prime}, \varphi^{\prime}\right)$ are the corresponding Euler angles for different molecules. $^{12}$

Further on, it is convenient to consider the rotations $\left(\psi, \theta^{\prime}, \varphi^{\prime}\right)$ in two stages where the intermediate orientation of the coordinate frame is characterized by the $z$ axis, parallel to the external field $\vec{B}$, i.e.,

$$
D_{\mu \mu^{\prime}}^{1}\left(\psi, \theta^{\prime}, \varphi^{\prime}\right)=\sum_{\mu_{0}} D_{\mu \mu_{0}}^{1}\left(\varphi_{0}, \theta_{0}, 0\right) D_{\mu_{0} \mu^{\prime}}^{1}(\psi,-\theta,-\varphi),
$$

with the polar and azimuthal angles $\theta_{0}, \varphi_{0}$, and $\theta, \varphi$ of the vector $\vec{B}$ in the laboratory and molecular frames, respectively. An explicit integration over the angle $\psi$ can be carried out in Eq. (12) as both the transition frequencies $\omega_{n}$ and the coefficients $C_{i m_{0}}, C_{f m_{e}}$ depend on the angles $\theta$ and $\varphi$ only $\left[\omega_{n}=\omega_{f}(l, \theta, \varphi)-\omega_{i}(l, \theta, \varphi) ; \quad C_{i m_{0}} \equiv C_{i m_{0}}(l, \theta, \varphi), \quad C_{f m_{e}}\right.$ $\left.\equiv C_{f m_{e}}(l, \theta, \varphi)\right]$.
Equation (12) involves an averaging procedure over the electronic multiplet states by summation over $l$, where the weight $w_{l}$ is determined by Eq. (5) and thus accounts for the slow relaxation of this multiplet. In the case of fast relaxation $\left[w_{l}=(2 S+1)^{-1}\right]$ the summation over $l$ in Eq. (12) may be omitted as according to Eqs. (4) and (5) the transition frequencies $\omega_{n}$ and the transition matrix elements no longer depend on this index.

Summarizing Eqs. (12)-(16) the following relations are derived:

$$
\begin{aligned}
U^{(j)}(\omega, d) \equiv & U^{(j)}\left(\omega, d, \theta_{0}\right) \\
= & \left\{\bar{U}+U_{01} \sin ^{2} \theta_{0}+(-1)^{j}\right. \\
& \left.\times\left(U_{01}^{2} \sin ^{4} \theta_{0}+U_{02}^{2} \cos ^{2} \theta_{0}\right)^{1 / 2}\right\} \\
\tilde{\rho}_{x x}^{(j)}(\omega)= & (1 / 2)\left[1-(-1)^{j} U_{01} \cos 2 \varphi_{0} \sin ^{2} \theta_{0},\right. \\
\times & \left.\left(U_{01}^{2} \sin ^{4} \theta_{0}+U_{02}^{2} \cos ^{2} \theta_{0}\right)^{-1 / 2}\right],
\end{aligned}
$$

and

$$
\begin{aligned}
\tilde{\rho}_{y x}^{(j)}(\omega)= & \frac{(-1)^{j}}{2}\left(U_{01} \sin 2 \varphi_{0} \sin ^{2} \theta_{0}-i U_{02} \cos \theta_{0}\right) \\
& \times\left(U_{01}^{2} \sin ^{4} \theta_{0}+U_{02}^{2} \cos ^{2} \theta_{0}\right)^{-1 / 2},
\end{aligned}
$$

with $\bar{U}=U_{1}+U_{-1}, U_{01}=U_{0}-\bar{U} / 2, U_{02}=U_{1}-U_{-1}$,

$$
\begin{aligned}
U_{\mu}(\omega, d)= & b(4 \pi)^{-1} \int_{0}^{\pi} \sin \theta d \theta \int_{0}^{2 \pi} d \varphi \sum_{l} w_{l}(\theta, \varphi) \\
& \times \sum_{i, f} w_{i f ; \mu}^{(l)}(\theta, \varphi) \\
& \times\left[\omega_{r}-\omega+\omega_{f}(\theta, \varphi ; l)-\omega_{i}(\theta, \varphi ; l)-i \Gamma / 2\right]^{-1},
\end{aligned}
$$

and

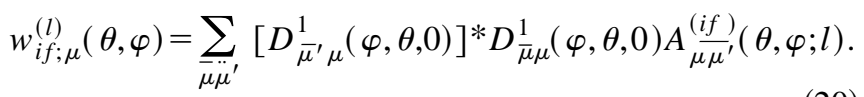

\section{NUMERICAL CALCULATIONS: ${ }^{57} \mathrm{Fe}$ MÖSSBAUER ABSORBERS}

In the previous section it has been shown that the amplitudes of forward-scattered SR can be expressed as

$$
\begin{aligned}
E_{\alpha}(0, \tau)= & \exp \left\{i\left[n_{\mathrm{el}}\left(\omega_{r}\right)\right] \omega d / c_{0}\right\} \sum_{j=1,2} \tilde{\rho}_{\alpha x}^{(j)}\left(\varphi_{0}\right) \chi_{0}(2 \pi)^{-1} \\
& \times \int_{-\infty}^{\infty} d \omega \exp (-i \omega \tau)\left\{\exp \left[i U^{(j)}\left(\omega, d, \theta_{0}\right)\right]-1\right\},
\end{aligned}
$$

with $\tau=t-d / c_{0}$. It has been assumed that the transient processes in the fast electronic subsystem have been terminated and a stationary polarization has been set in for all actual values of $\tau$. Therefore $n_{\mathrm{el}}(\omega)$ has been considered as a constant in Eq. (21). 
Equation (21) together with Eqs. (14) and (17)-(20) form a complete basis for simulations of the time-domain spectra of forward-scattered SR if the eigenfunctions and the eigenvalues of the Hamiltonians (1) and (2) are known. The FORTRAN program SYNFOS, which has been written in order to verify the correct formulation of the theory, carries out the numerical integrations over the frequency parameter $\omega$ and angles $\theta, \varphi$ in Eqs. (17) and (21), respectively, and diagonalizes the Hamiltonians (1) and (2). The output of the program presents the time-dependent intensity of the modified forward-scattered pulse:

$$
i(t)=|\vec{E}(0, t)|^{2} \exp (2 \mu d) /\left|\chi_{0}\right|^{2} .
$$

$2 \mu$ is the coefficient of nonresonant absorption with $\mu$ $=\operatorname{Im} n_{\mathrm{el}}\left(\omega_{r}\right)$.

The integration over Euler angles $\theta$ and $\varphi$ is performed using a set of grid points which are to a high degree of approximation homogeneously distributed over an octant of the unit sphere. ${ }^{13}$ The number of grid points per octant is 6 $\times 2^{n}$ where $n$ can be chosen from 2 to 7 , while the number of octants to be evaluated is determined by the symmetry of the actual system. The field potentials $\vec{E}(0, t)$ have been treated as the sum of two additives:

$$
\vec{E}(0, t)=\vec{E}^{\prime}(0, t)+\vec{E}^{\prime \prime}(0, t)
$$

where

$$
\begin{aligned}
& E_{\alpha}^{\prime}(0, t)=\chi_{0} \Theta(t) \sum_{\mu=0, \pm 1} \zeta_{\alpha \mu}\left(\theta_{0}, \varphi_{0}\right) U_{\mu}(t, d), \\
& U_{\mu}(t, d)=-(4 \pi)^{-1} b \exp \left[\left(i n_{\mathrm{el}} \omega d / c_{0}\right)-(\Gamma t / 2)\right] \\
& \times \int_{0}^{\pi} \sin \theta d \theta \int_{0}^{\pi} d \varphi \sum_{l} w_{l} \\
& \quad \times \sum_{i, f}\left\{\sum_{j=1,2} \tilde{\rho}_{\alpha x}^{(j)}\left(\varphi_{0}\right) w_{i f ; \mu}^{(l)}\right. \\
&\left.\times \exp \left[-i\left(\omega_{r}+\omega_{f}-\omega_{i}\right) t\right]\right\}
\end{aligned}
$$

the coefficients $\zeta_{\alpha \mu}$ depend on angular variables, and

$$
\begin{aligned}
E_{\alpha}^{\prime \prime}(0, t)= & \chi_{0} \exp \left(i n_{\mathrm{el}} \omega d / c_{0}\right) \sum_{j=1,2} \tilde{\rho}_{\alpha x}^{(j)}\left(\varphi_{0}\right)(2 \pi)^{-1} \\
& \times \int_{-\omega_{M}+\omega_{r}}^{\omega_{M}+\omega_{r}} d \omega \exp (-i \omega t)\left\{\exp \left[i U^{(j)}\left(\omega, d, \theta_{0}\right)\right]\right. \\
& \left.-1-i U^{(j)}\left(\omega, d, \theta_{0}\right)\right\}
\end{aligned}
$$

The integrand in Eq. (25) decreases as $\left|\omega-\omega_{r}\right|^{-2}$, if $\left|\omega-\omega_{r}\right| \rightarrow \infty$. Therefore a frequency interval of reasonable length should be chosen to achieve sufficient precision of the approximate value of the original infinite integral, calculated as a fast Fourier transform. Examples of the calculated spectra are represented in the following sections.

\section{CALCULATED MÖSSBAUER SPECTRA IN THE TIME DOMAIN}

From the first few experiments on powder samples reported in the literature, ${ }^{14-16}$ it has become already quite obvious that the interpretation of time-dependent forward scattering is not as straightforward as with conventional Mössbauer absorption spectra. The reason is that it seems to be impossible to discriminate more than two frequencies in a beating pattern by simple viewing, so that only in the case where one deals with a single species of absorbing atoms in the sample one may distinguish between a magnetic dipole and an electric quadrupole interaction by mere visual inspection. Ambiguities show up already when, e.g., two species with different quadrupole interactions are present. While this can be recognized in general easily at the first glance from the appearance of additional absorption peaks in the conventional spectra in the energy domain, spectroscopy in the time domain contains such information only in the special interference pattern. Such ambiguities can only be resolved by a detailed numerical analysis on the basis of a carefully worked-out theory.

This situation pretends to look at the first glance as a serious drawback of an otherwise promising novel spectroscopy. But nevertheless great expectations rest on Mössbauer spectroscopy in the time domain especially for biological applications, because it promises to be more sensitive to hyperfine parameters when the measurements are performed to high enough delay times, and because of its obvious applicability to much smaller samples sizes. In the following the results of calculations with the SYNFOS program will be shown in order to elucidate the aspects mentioned above. The actual calculations were performed with a parameter set taken from a system, which possesses relevance in the field of bioinorganic chemistry and which might serve therefore as a suitable test case.

\section{A. Zero-field spectra of a ferrous high-spin complex}

The Mössbauer spectroscopic properties of the ferrous high-spin state $\left(3 d^{6}, \quad S=2\right)$ of the complex $\left[\mathrm{Fe}\left(\mathrm{CH}_{3} \mathrm{COO}\right)\left(\mathrm{TP}_{\text {piv }} \mathrm{P}\right)\right]^{-}$, which is a biomimetic analog of the prosthetic group P460 of the multiheme enzyme hydroxylamine oxidoreductase from the bacterium Nitrosomonas europeae, are well known from field- and temperaturedependent measurements together with spin-Hamiltonian simulations in the energy domain. ${ }^{17}$ Remarkable is the large nearly-temperature-independent quadrupole splitting of about $+4.2 \mathrm{~mm} \mathrm{~s}^{-1}$ originating from an "excess" electron beyond the half-filled $3 d$ shell in an oblate $d_{x y}$ orbital. In Figs. 1(a) and 1(b) the time spectra for two effective thicknesses $T_{M}$ are reproduced, indicating the presence of quantum beats with frequency $\omega=\Delta E_{Q} / \hbar$ and the appearance of additional dynamical beats with increasing effective thickness. Practically the same beat pattern as for $T_{M}=10, \Delta E_{Q}$ $=4.2 \mathrm{~mm} \mathrm{~s}^{-1}$ [Fig. 1(a)] is obtained for a sample with $T_{M}$ $=4$ and a 1:1 mixture of two species with quadrupole splittings 4.0 and $4.4 \mathrm{~mm} \mathrm{~s}^{-1}$, respectively [Fig. 1(c)]. We have the corresponding situation when comparing $T_{M}=40, \Delta E_{Q}$ $=4.2 \mathrm{~mm} \mathrm{~s}^{-1} \quad\left[\right.$ Fig. 1(b)] with $T_{M}=4, \quad \Delta E_{Q}$ $=3.8 / 4.6 \mathrm{~mm} \mathrm{~s}^{-1}$ [Fig. 1(d)]. In both respective pairs of spectra $[(a),(c)]$ and $[(b),(d)]$, minima due to destructive in- 

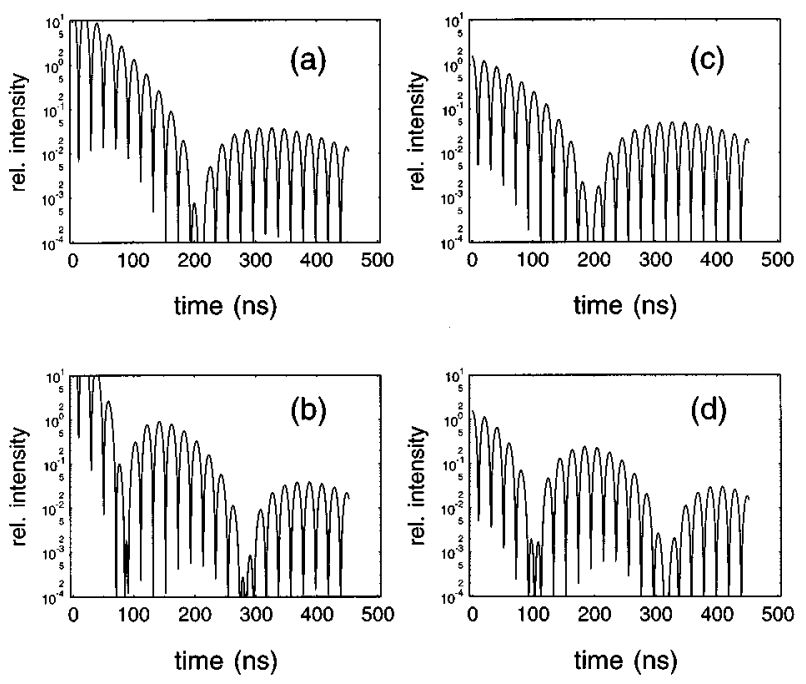

FIG. 1. Calculated time spectra for absorbers showing pure quadrupole splitting: (a) $\Delta E_{Q}=4.2 \mathrm{~mm} \mathrm{~s}^{-1}, T_{M}=10$, (b) $\Delta E_{Q}$ $=4.2 \mathrm{~mm} \mathrm{~s}^{-1}, T_{M}=40$, (c) a $1: 1$ mixture of $\Delta E_{Q}=4.0$ and $4.4 \mathrm{~mm} \mathrm{~s}^{-1}, T_{M}=4$, and (d) a $1: 1$ mixture of $\Delta E_{Q}=3.8$ and $4.6 \mathrm{~mm} \mathrm{~s}^{-1}, T_{M}=4$.

terferences appear at about the same delay times. To resolve this ambiguity it is necessary to perform measurements with different effective thicknesses, e.g., by recording temperature-dependent spectra of the sample, or to estimate $T_{M}$ separately.

An additional example for coherent superposition of two quadrupole splittings in the time domain is provided by Fig. 2. The effective thickness is $T_{M}=5$, and the 1:1 mixture consists of two species, one with a $3 d^{6} ; S=2$ configuration and $\Delta E_{Q, 1}=4.2 \mathrm{~mm} \mathrm{~s}^{-1}$, and the other with a $3 d^{5}, S=5 / 2$ configuration and $\Delta E_{Q, 2}=0.55 \mathrm{~mm} \mathrm{~s}^{-1}$; additionally, the difference of isomer shifts is $\delta_{1}-\delta_{2}=0.65 \mathrm{~mm} \mathrm{~s}^{-1}$. This example demonstrates that a simple case like the 1:1 superposition of two quadrupole splittings, which could easily be recognized by mere viewing of the spectrum in the energy domain, cannot be analyzed in the time domain without numerical analysis.

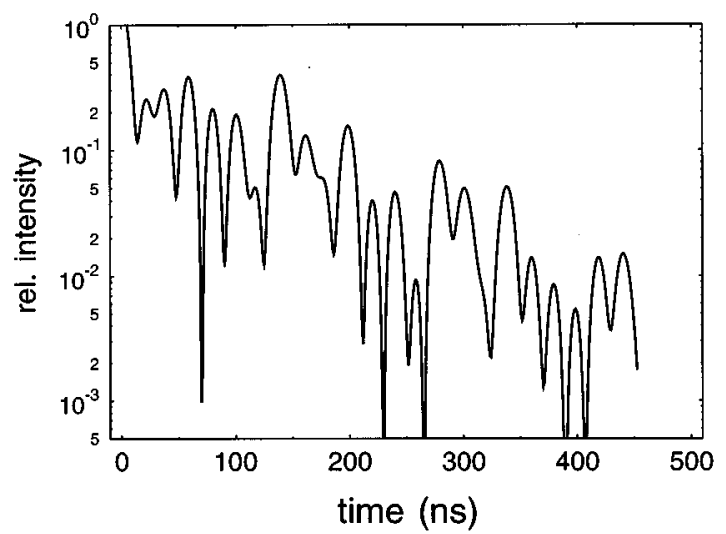

FIG. 2. Calculated time spectrum for a 1:1 mixture of two quadrupole split species, one with $\Delta E_{Q, 1}=4.2 \mathrm{~mm} \mathrm{~s}^{-1}$ and the other with $\Delta E_{Q, 2}=0.55 \mathrm{~mm} \mathrm{~s}^{-1}$ and with a difference of isomer shifts of $\delta_{1}-\delta_{2}=0.65 \mathrm{~mm} \mathrm{~s}^{-1}, T_{M}=5$.

\section{B. $\alpha$-iron}

Ferromagnetically ordered metallic iron ( $\alpha$-iron) is, because of its characteristic magnetic hyperfine splitting, commonly used for calibrating Mössbauer spectra in the energy domain. For this purpose it is rather irrelevant whether or not the sample foil is magnetized by a small external field, i.e., whether the magnetic moments of the domains are oriented along the field or are oriented randomly, because this influences only the intensities of the absorption lines and not their positions. In NFS this, however, makes a significant difference: Since the beating of the forward-scattering pattern is due to coherent excitation of a multitude of nuclear resonances, whose scattering amplitudes depend strongly on their respective magnetic quantum numbers, the effect of magnetization changes the beating pattern completely. In the unmagnetized case (with the preferred orientation of the mag-
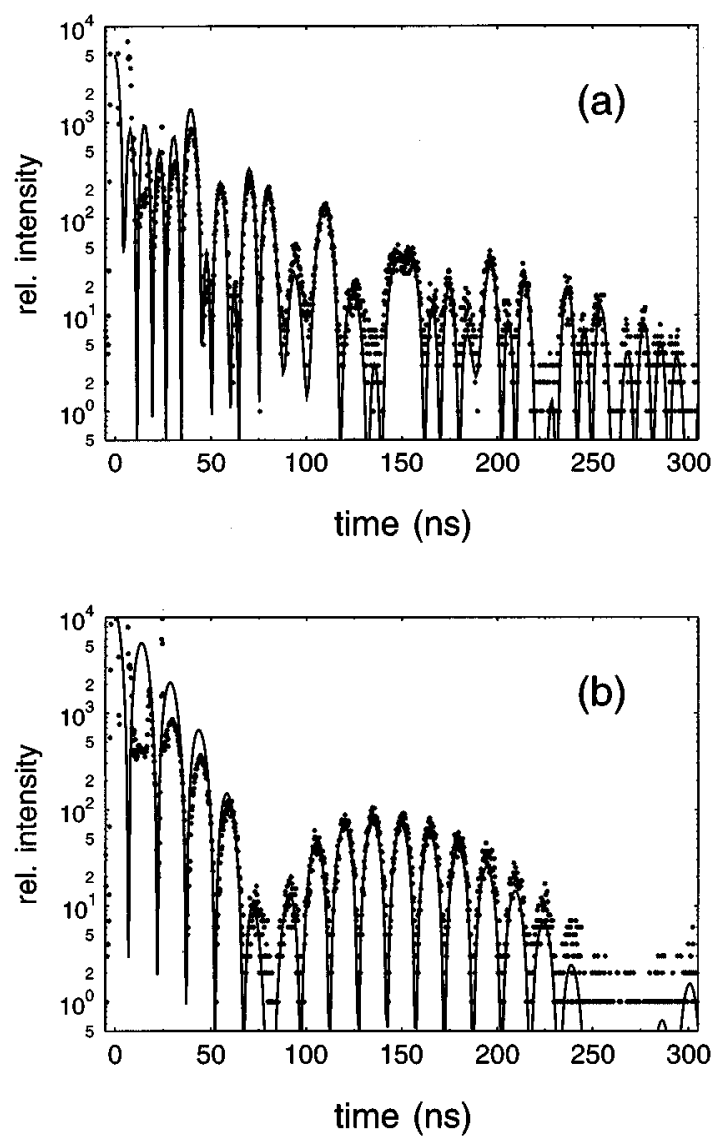

FIG. 3. Time spectra from a 3- $\mu$ m-thick $\alpha-{ }^{57} \mathrm{Fe}$ foil at $4.2 \mathrm{~K}$, (a) unmagnetized and (b) fully magnetized by an external field $B$ $=3 \mathrm{~T}$ applied perpendicular to $\vec{\kappa}$ and $\vec{\sigma}$. The solid lines represent simulations by means of SYNFOS with $T_{M}=52$ and accounting for the fact that in the so-called unmagnetized case the magnetization exhibits preferred orientation towards the plane of the foil, which is perpendicular to $\vec{\kappa}$; i.e., the magnetization is randomly distributed within orientations, which decline by $\leqslant 45^{\circ}$ from the plane of the foil. To account for the correct hyperfine field of $-33.75 \mathrm{~T}$, the electronic state of iron is taken as $S=3 / 2$, the hyperfine field per unit spin as $A_{x, y, z} / g_{n} \beta_{n}=-22.5 \mathrm{~T}$, the zero field splitting as $D$ $=0 \mathrm{~cm}^{-1}$, and the formal temperature as low as $1 \mathrm{mK}$. Under these conditions the hyperfine interaction produced by the $\pm 3 / 2$ Kramers doublet in slow relaxation is in the spin-Hamiltonian formalism identical to that of $\alpha$-iron. 
netization towards the plane of the foil), a large number of resonant lines, which couple to the radiation field, yield a complicated pattern of superimposed quantum beats [Fig. 3(a)] compared to the fully magnetized case with a field $\vec{B}$ applied perpendicular to $\vec{\kappa}$ and $\vec{\sigma}$, i.e., parallel to the magnetic vector of the incident radiation, where only quantum beats between the two $\Delta m=0$ lines of the nuclear levels of ${ }^{57} \mathrm{Fe}$ appear [Fig. 3(b)].

\section{Magnetic hyperfine spectra of a ferrous high-spin complex in an applied field}

Application of an external field $\vec{B}$ to a ferrous high-spin complex, in the present case a polycrystalline sample of
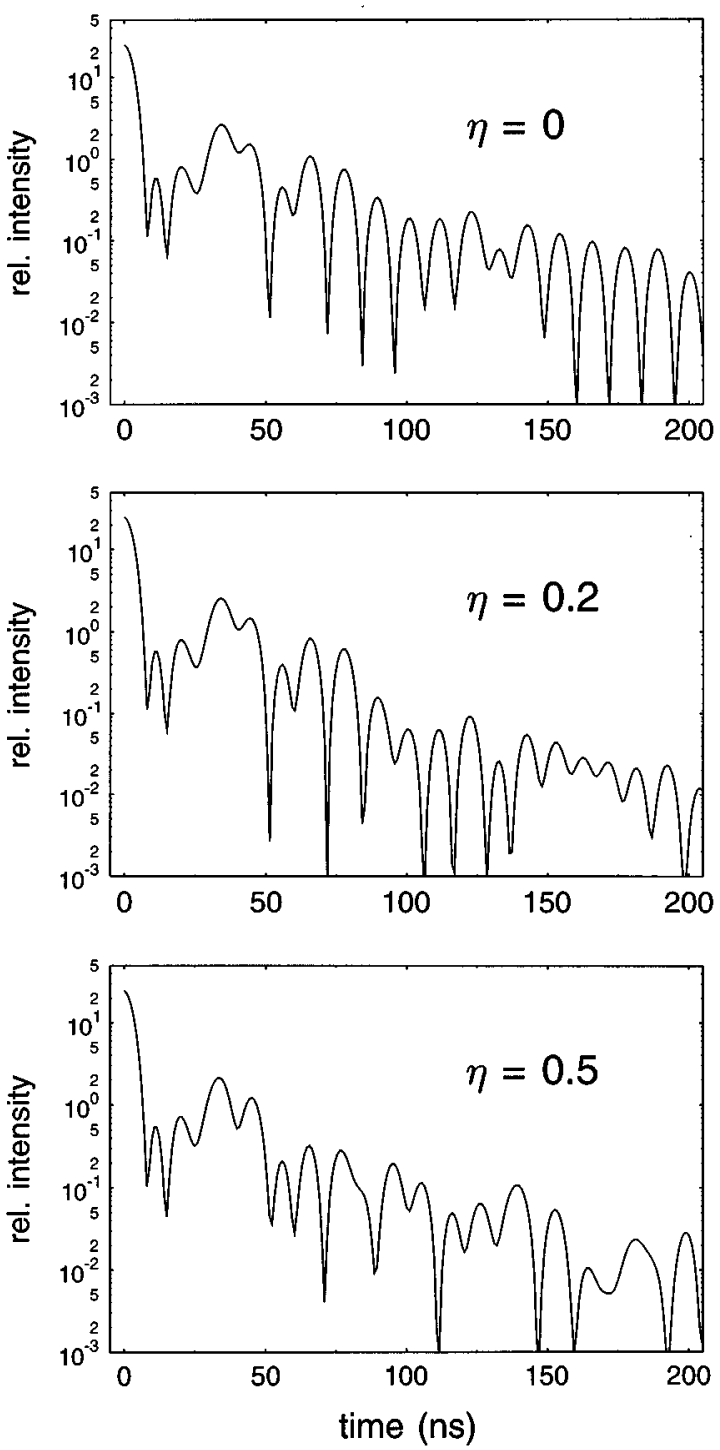

FIG. 4. Time spectra for a ferrous high-spin system in an external field $B=6 \mathrm{~T}$ applied perpendicular to $\vec{\kappa}$ and $\vec{\sigma}$ of the incoming beam calculated for a quadrupole splitting $\Delta E_{Q}=4.25 \mathrm{~mm} \mathrm{~s}^{-1}$ with asymmetry parameter $\eta$ taken as $0,0.2$, and 0.5 as indicated. The values of the other relevant parameters are $D=-0.8 \mathrm{~cm}^{-1}, E / D$ $=0$ for the zero-field splitting and $A_{x, y} / g_{n} \beta_{n}=-17 \mathrm{~T}, A_{z} / g_{n} \beta_{n}$ $=-12 \mathrm{~T}$ for the magnetic hyperfine coupling tensor, $T_{M}=20$. Slow relaxation at a temperature $T=3.3 \mathrm{~K}$ was assumed.
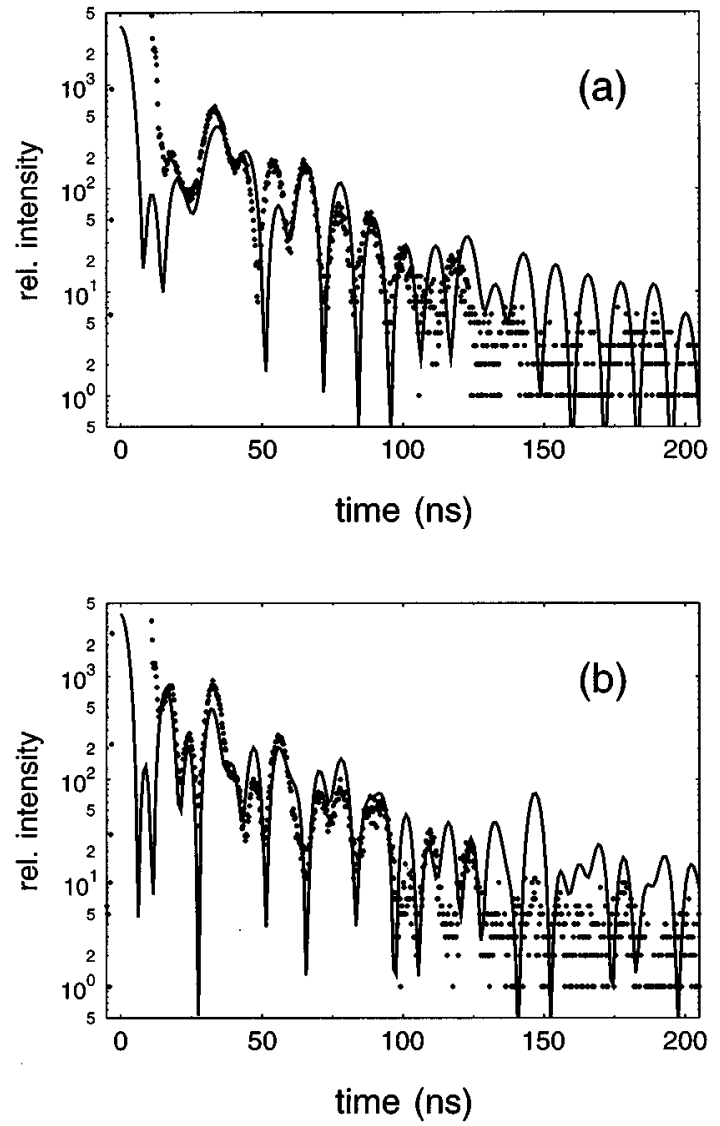

FIG. 5. Measured time spectra from $\left[\mathrm{Fe}^{\mathrm{II}}\left(\mathrm{CH}_{3} \mathrm{COO}\right) \mathrm{TP}_{\text {piv }} \mathrm{P}\right]^{-}$at $3.3 \mathrm{~K}$ in an external field of $6.0 \mathrm{~T}$ applied (a) perpendicular to $\vec{\kappa}$ and $\vec{\sigma}$ and (b) perpendicular to $\vec{\kappa}$ and parallel to $\vec{\sigma}$ of the incoming beam (dots). The solid lines are calculations by means of SYNFOS using $\eta=0$ and the spin-Hamiltonian parameters given underneath Fig. 4. Only a common normalization factor has been applied and a representative background of 0.5 counts added.

$\left[\mathrm{Fe}\left(\mathrm{CH}_{3} \mathrm{COO}\right)\left(\mathrm{TP}_{\text {piv }} \mathrm{P}\right)\right]^{-}$, induces spin-expectation values according to Eqs. (1) and (3), which depend on the polar and azimuthal angles $\theta$ and $\varphi$, respectively, of $\vec{B}$ with respect to the molecular frame of reference. Accordingly, the magnetic hyperfine splitting of the nuclear levels, Eq. (2), is characterized for the randomly oriented paramagnetic sample by a complicated band of resonant absorptions. $\left[\mathrm{Fe}\left(\mathrm{CH}_{3} \mathrm{COO}\right)\left(\mathrm{TP}_{\text {piv }} \mathrm{P}\right)\right]^{-}$therefore is thought to serve as a test case for comparing measured NFS spectra with those which result from a SYNFOS simulation of a specific random distribution of hyperfine patterns. The specificity of this distribution is defined by the spin-Hamiltonian parameters of $\left[\mathrm{Fe}\left(\mathrm{CH}_{3} \mathrm{COO}\right)\left(\mathrm{TP}_{\text {piv }} \mathrm{P}\right)\right]^{-}$, which are $D=-0.8 \mathrm{~cm}^{-1}, E / D$ $=0$ for the zero-field splitting, and $A_{x, y} / g_{n} \beta_{n}=-17 \mathrm{~T}$, $A_{z} / g_{n} \beta_{n}=-12 \mathrm{~T}$ for the magnetic hyperfine coupling parameters. ${ }^{16}$ Figure 4 gives the results of SYNFOS calculations for a randomly oriented absorber with $T_{M}=20$, temperature $T=3.3 \mathrm{~K}$, and external field $B=6 \mathrm{~T}$ applied perpendicular to $\vec{\kappa}$ and $\vec{\sigma}$ of the synchrotron beam. The time spectra for $\Delta E_{Q}=4.25 \mathrm{~mm} \mathrm{~s}^{-1}$ and $\eta=0,0.2$, and 0.5 as indicated are reproduced to demonstrate the sensitivity of the timedependent forward scattering on this parameter. Comparable 
sensitivity is achieved for other parameters, provided the measurements are performed to high enough delay times.

In Fig. 5 experimental NFS spectra of $\left[\mathrm{Fe}\left(\mathrm{CH}_{3} \mathrm{COO}\right)\left(\mathrm{TP}_{\text {piv }} \mathrm{P}\right)\right]^{-}$are shown, which were recorded at $3.3 \mathrm{~K}$ in an external field of $6.0 \mathrm{~T}$ applied perpendicular to $\vec{\kappa}$ and $\vec{\sigma}$ and perpendicular to $\vec{\kappa}$ and parallel to $\vec{\sigma}$ of the incoming beam. Details of the experimental setup of the Mösbauer beam line at HASYLAB, Hamburg, where the measurements have been performed, are described elsewhere. ${ }^{18}$ Note that the sample had very high effective thickness $\left(T_{M}\right.$ $=20$ ) in order to guarantee reasonable statistics at least up to a delay time of $\sim 150 \mathrm{~ns}$. Any inhomogeneity, e.g., any distribution in $T_{M}$, which very likely occurs in a polycrystalline sample with such high average effective thickness, would affect the dynamical beat structure of the NFS spectra. Even without the possible improvement due to a distribution in $T_{M}$ the solid lines in Fig. 5, which represent SYNFOS simulations with the parameters from above and with $T_{M}=20$, reflect the main features of the measured NFS spectra.

In an actual attempt to unambiguously derive Mössbauer and spin-Hamiltonian parameters from a SYNFOS analysis of experimental NFS spectra, a number of different experimental conditions (temperature, magnitude, and direction of the applied field) have to be met. In this respect the Mössbauer spectroscopies in the energy and time domains are comparable.

\section{CONCLUSION}

Starting from the first principles of $\gamma$ optics, the timedependent scattering of randomly oriented molecules was not considered before, and therefore the basic formulas from the published work on $\gamma$ optics ${ }^{5-7}$ could not directly be used. For this reason we have shown here how the basic formulas are derived for the description of this problem. The analysis of NFS spectra by simple viewing is restricted to samples which have a small effective thickness $\left(T_{M}<1\right)$ and which exhibit a single-beat frequency only. Otherwise, a numerical analysis of the spectra is inevitable.

The program package SYNFOS has been developed to take into account two aspects, which are of particular importance when dealing with randomly oriented paramagnetic absorbers: (i) It evaluates numerically the basic formulas which adequately describe the time-dependent scattering of these absorbers, and (ii) it applies the spin-Hamiltonian formalism which accounts for their magnetic properties. From the calculated spectra of the two examples shown, $\left[\mathrm{Fe}\left(\mathrm{CH}_{3} \mathrm{COO}\right)\left(\mathrm{TP}_{\text {piv }}\right) \mathrm{P}\right]^{-}$and $\alpha-\mathrm{Fe}$, it appears that hyperfine and spin-Hamiltonian parameters can in principle be determined in the time domain with much higher accuracy than in the energy domain if the rate of the delayed counts is followed far enough in time.

In a forthcoming paper we shall describe applications of SYNFOS with temperature- and field-dependent simulations of NFS spectra including slow, intermediate, and fast electronic relaxation of paramagnetic systems.

\section{ACKNOWLEDGMENT}

This work was supported by the BMBF Grant Nos. 05 5FLAXI 4 and 05643 FLA 3.
${ }^{1}$ J. B. Hastings, D. P. Siddons, U. van Bürck, R. Hollatz, and U. Bergmann, Phys. Rev. Lett. 66, 770 (1991).

${ }^{2}$ E. Gerdau and U. van Bürck, in Resonant Anomalous X-Ray Scattering, edited by G. Materlik, C. J. Sparks, and U. Fischer (Elsevier, New York, 1994), p. 589.

${ }^{3}$ W. Sturhahn and E. Gerdau, Phys. Rev. B 49, 9285 (1994).

${ }^{4}$ L. Deak, L. Bottyan, D. L. Nagy, and H. Spiering, Phys. Rev. B 53, 6158 (1996).

${ }^{5}$ A. M. Afanasiev and Yu. Kagan, Zh. Eksp. Teor. Fiz. 48, 327 (1965) [Sov. Phys. JETP 21, 215 (1965)].

${ }^{6}$ J. P. Hannon and G. T. Trammel, Phys. Rev. 169, 315 (1968).

${ }^{7}$ J. P. Hannon and G. T. Trammel, Phys. Rev. 186, 306 (1969).

${ }^{8}$ A. Abragam and M. H. L. Pryce, Proc. R. Soc. London, Ser. A 205, 135 (1951).

${ }^{9}$ A. X. Trautwein, E. Bill, E. L. Bominaar, and H. Winkler, Struct. Bond. 78, 1 (1991).

${ }^{10}$ Yu. Kagan, A. M. Afanasiev, and V. G. Kohn, J. Phys. C 12, 615 (1979).

${ }^{11}$ M. Blume and O. C. Kistner, Phys. Rev. 171, 417 (1968).
${ }^{12}$ R. A. Edmonds, Angular Momentum in Quantum Mechanics (Princeton University Press, Princeton, NJ, 1957).

${ }^{13}$ K. Knese, Ph.D. thesis, Universität Leipzig, 1995.

${ }^{14}$ E. E. Alp, W. Sturhahn, and T. Toellner, Nucl. Instrum. Methods Phys. Res. B 97, 526 (1995).

${ }^{15}$ O. Leupold, H. Grünsteudel, W. Meyer, H. F. Grünsteudel, H. Winkler, D. Mandon, H. D. Rüter, J. Metge, E. Realo, E. Gerdau, A. X. Trautwein, and R. Weiss, in Conference Proceedings Italian Physical Society Vol. 50, ICAME-95, edited by I. Ortalli (SIF, Bologna, 1996), p. 857.

${ }^{16}$ E. Realo, M. Haas, W. Meyer, O. Leupold, H. Winkler, H. Grünsteudel, H. F. Grünsteudel, J. Metge, E. Gerdau, and A. X. Trautwein, in ICAME-95 (Ref. 15), p. 861.

${ }^{17}$ E. L. Bominaar, X.-Q. Ding, A. Gismelseed, E. Bill, H. Winkler, A. X. Trautwein, H. Nasri, J. Fischer, and R. Weiss, Inorg. Chem. 31, 1845 (1992).

${ }^{18}$ O. Leupold, E. Gerdau, H. D. Rüter, W. Meyer-Klaucke, A. X. Trautwein, and H. Winkler (unpublished). 